\title{
Persistent postoperative limitation of range of motion after total knee arthroplasty - Are there predictors?
}

Stella Oberberg ( $\nabla$ stella.oberberg@mlk-bochum.de)

Katholisches Klinikum Bochum Sankt Josef-Hospital https://orcid.org/0000-0002-3761-1986

Jessica Dammers

Katholisches Klinikum Bochum Sankt Josef-Hospital

Jan Nottenkämper

Katholisches Klinikum Bochum Sankt Josef-Hospital

Jan Krapp

Katholisches Klinikum Bochum Sankt Josef-Hospital

Roland Ernst Willburger

Katholisches Klinikum Bochum Sankt Josef-Hospital

\section{Research Article}

Keywords: total knee arthroplasty, arthrofibrosis, range of motion, complications after total knee arthroplasty, outcome

Posted Date: March 13th, 2021

DOl: https://doi.org/10.21203/rs.3.rs-299870/v1

License: (c) (i) This work is licensed under a Creative Commons Attribution 4.0 International License. Read Full License 


\section{Abstract}

\section{Background}

Implantation of total knee arthroplasty (TKA) is one of the most common operations in orthopedics. However, about $20 \%$ of all patients are not satisfied with the result after TKA. Thus, persistent postoperative limitation of range of motion, for instance in the context of arthrofibrosis is with approximately $10 \%$ one of the most frequent complications after TKA and, thus, one of the most reasons for an operative revision or a worse postoperative outcome. Some potential therapeutic strategies are described in recent literature including patient-related risk factors and models for the pathogenesis of arthrofibrosis. The weighting of individual risk factors is currently not fully understood.

\section{Methods}

In the present study, a retrospective analysis of patient data was performed using a literature-based questionnaire in 72 patients following mobilization under anesthesia due to persistent limitation of range of motion (ROM) after TKA. An existing internal data set of 72 patients without restriction of ROM after TKA was used as a control-population. The study-group was invited to a follow-up examination to assess long-term outcome by several scores "Knee osteoarthritis outcome score" (KOOS) and "Knee society score" (KSS). In addition, patient expectation and satisfaction were evaluated according to the New KSS.

Results

In total, 144 patients were included in the retrospective analysis. Person-related risk factors described in the literature, such as gender or secondary diseases (diabetes mellitus, rheumatoid arthritis, or psychiatric diseases) could not be confirmed as risk factors in our population. There was a statistically significant difference in pre- and postoperative range of motion. The study-group showed a essentially higher preoperative flexion deficit and developed a lower postoperative range of motion in both extension and flexion deficits. Significant differences were found with respect to previous surgeries and duration of surgery. The study-population showed an increased number of previous operations, especially arthroscopic surgeries of the knee joint, as well as a prolonged operation time exceeding 90 minutes. Preoperative patient expectations were not fulfilled on average, despite this, patient satisfaction was still high in the long term examination.

\section{Conclusions}

We determined risk factors for our population. For example, the preoperative range of motion, previous operations, and the duration of surgery were relevant factors that were closely related to the postoperative outcome of our study-population. However, due to the retrospective study design, these results are limited. In order to perform an exact evaluation of the risk factors mentioned above, we would recommend to supplement a prospective investigation with regard to these aspects. In the follow-up examination, we furthermore could confirm the significant impact of patient expectation on outcome after TKA as described in the literature. 


\section{Full Text}

Due to technical limitations, full-text HTML conversion of this manuscript could not be completed. However, the manuscript can be downloaded and accessed as a PDF.

\section{Figures}

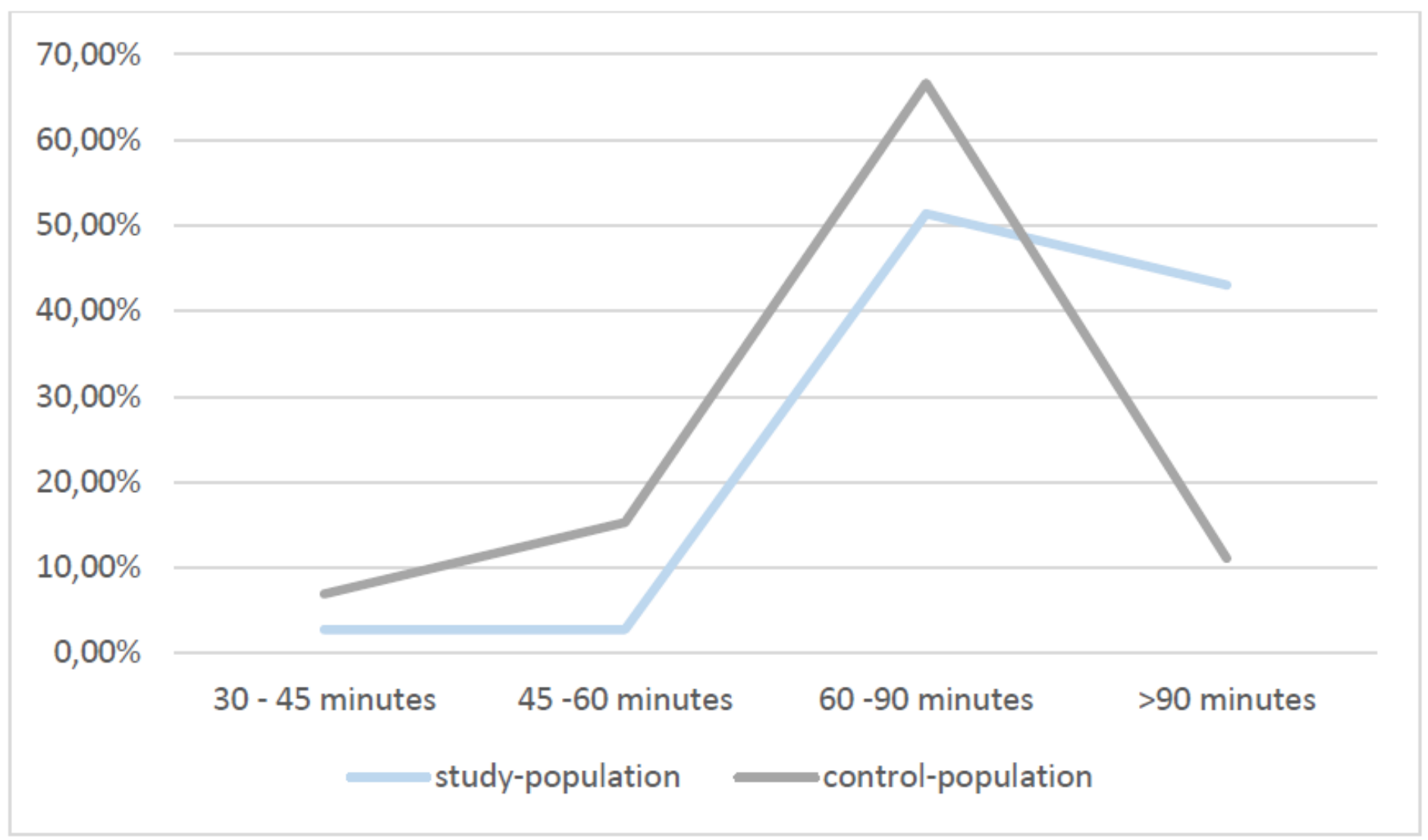

\section{Figure 1}

Duration of surgery compared between the populations. 


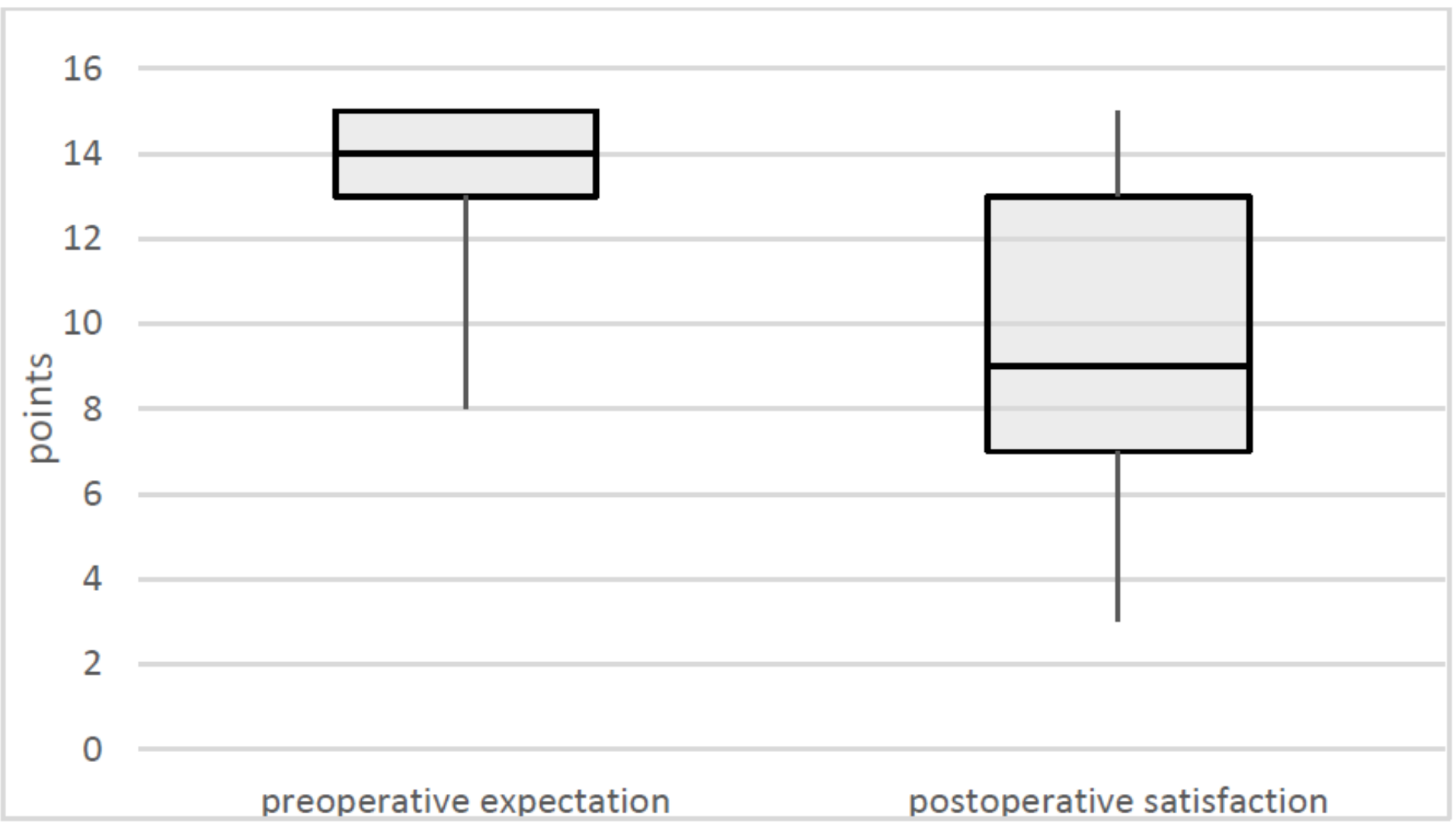

Figure 2

Preoperative patient expectation and postoperative patient satisfaction at follow-up

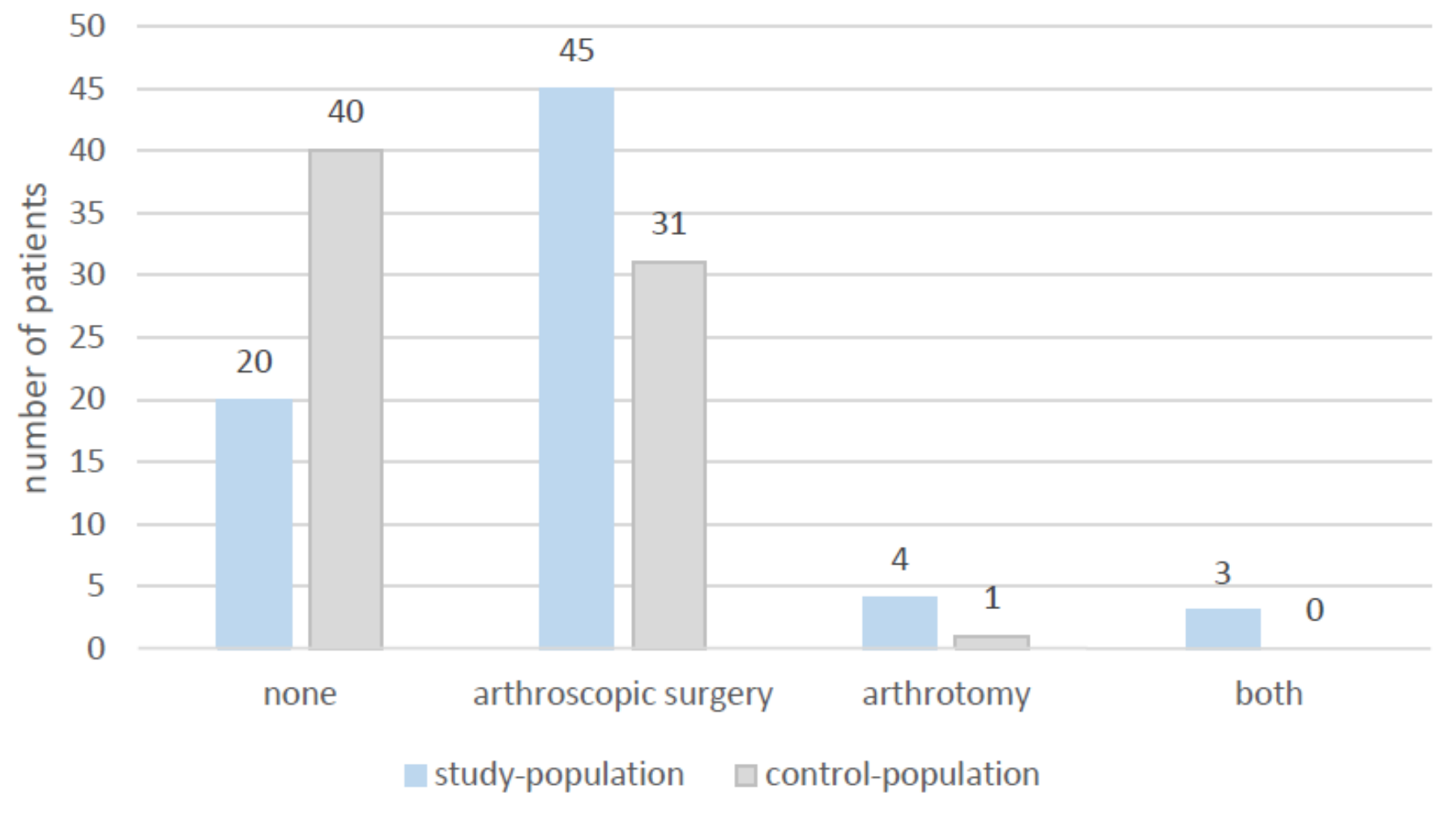


Figure 3

Comparison of previous operations of the study-population and the control-population 